\title{
Antibacterial effect evaluation of moxalactam
} against extended-spectrum $\beta$-lactamase-producing Escherichia coli and Klebsiella pneumoniae with in vitro pharmacokinetics/pharmacodynamics
simulation

This article was published in the following Dove Press journal: Infection and Drug Resistance

\section{Chen Huang ${ }^{1, *}$ \\ Beiwen Zhengl,* \\ Wei $\mathrm{Yu}^{2}$ \\ Tianshui Niu' \\ Tingting Xiao' \\ Jing Zhang' \\ Yonghong Xiao'}

'State Key Laboratory for Diagnosis and Treatment of Infectious Diseases, Collaborative Innovation Center for Diagnosis and Treatment of Infectious Diseases, The First Affiliated Hospital, College of Medicine,

Zhejiang University, Hangzhou, China; ${ }^{2}$ Department of Infectious Diseases, Zhejiang Provincial People's Hospital, Hangzhou, China

*These authors contributed equally to this work

Correspondence: Yonghong Xiao State Key Laboratory for Diagnosis and Treatment of Infectious Diseases, Collaborative Innovation Center for Diagnosis and Treatment of Infectious Diseases, The First Affiliated Hospital, College of Medicine, Zhejiang University, No 79 Qingchun Road, Hangzhou 310003, China

Tel/fax +86 5718723642 I

Email xiaoyonghong@zju.edu.cn
Objectives: The aim of this study was to evaluate the bactericidal effects of moxalactam (MOX), cefotaxime (CTX), and cefoperazone/sulbactam (CFZ/SBT) against extended-spectrum $\beta$-lactamase (ESBL) producing Escherichia coli and Klebsiella pneumoniae, using an in vitro pharmacokinetics (PK)/pharmacodynamics model.

Methods: Two clinical ESBL-producing strains ( bla $_{C T X-M-15}$ positive E. coli 3376 and bla $_{C T X-M-14}$ positive K. pneumoniae 2689) and E. coli American Type Culture Collection (ATCC)25922 were used in the study. The PK Auto Simulation System 400 was used to simulate the human PK procedures after intravenous administration of different doses of MOX, CTX, and CFZ/ SBT. Bacterial growth recovery time (RT) and the area between the control growth curve and bactericidal curves (IE) were employed to assess the antibacterial efficacies of all the agents. Results: The minimum inhibitory concentrations of MOX, CTX, and CFZ/SBT against E. coli ATCC25922, 3376, and 2689 strains were 0.5, 0.5, 0.25; 0.06, >256, 256; and 0.5/0.5, $16 / 16,32 / 32 \mathrm{mg} / \mathrm{L}$. All the agents demonstrated outstanding bactericidal effects against $E$. coli ATCC25922 (RT $>24 \mathrm{~h}$ and IE $>120 \log _{10} \mathrm{CFU} / \mathrm{mL} \cdot \mathrm{h}^{-1}$ ) with simulating PK procedures, especially in the multiple dose administration models. Against ESBL producers, CTX and CFZ/SBT displayed only weak bactericidal effects, and subsequent regrowth was evident. MOX exhibited potent antibacterial activity against all the strains tested. The values of effective parameters of MOX were much higher than those of CTX and CFZ/SBT (the bacterial RTs with the 3 agents were $>24,<4$, and $<13 \mathrm{~h}$, and the IEs were $>110,<10$, and $<60 \log _{10} \mathrm{CFU} / \mathrm{mL} \cdot \mathrm{h}^{-1}$, respectively). Conclusion: MOX demonstrated excellent bactericidal effect, which is worthy of further exploration to serve as an alternative therapeutic agent against ESBL-producing Enterobacteriaceae. Keywords: moxalactam, in vitro, pharmacokinetics/pharmacodynamics, extended-spectrum $\beta$-lactamases, Escherichia coli, Klebsiella pneumoniae

\section{Introduction}

A report from the World Health Organization revealed that the resistance of Escherichia coli and Klebsiella pneumoniae to third-generation cephalosporins is spreading. ${ }^{1}$ Extended-spectrum $\beta$-lactamases (ESBLs) confer resistance to most commonly used $\beta$-lactam agents, and often co-resistance to other antimicrobial classes, leading to the failure of clinical treatment. ${ }^{2}$ Carbapenems are recognized as the first-line therapeutic agents to manage ESBL-producing Enterobacteriaceae infections. ${ }^{2,3}$ However, 
the increased use of carbapenems may generate selection pressure for carbapenem-resistant isolates, resulting in their evolution and dissemination. Thus, the prudent use of antimicrobials in the clinic is crucial, as is finding alternatives of carbapenems to treat infections caused by ESBL-producing isolates and avoid exacerbation of the spreading of ESBLs.

Moxalactam (MOX) is a synthetic oxacephem $\beta$-lactam agent in which the sulfur atom in the cephalosporin nucleus is substituted by an oxygen atom, representing a 7a-methoxysubstitution. ${ }^{4}$ MOX displays potent in vitro antibacterial activity against ESBL-producing Enterobacteriaceae and has favorable pharmacological properties, including a high serum concentration and large apparent distribution volume. ${ }^{4}$ However, according to the descriptions of the Clinical and Laboratory Standards Institute (CLSI), ESBL-producing E. coli and K. pneumoniae should be reported as resistant to MOX irrespective of the minimum inhibitory concentration (MIC), because MOX has limited availability in many countries and clinical experience on its use in the treatment of ESBL-producing bacterial infections is scarce, and its effect is therefore controversial. ${ }^{5}$ In the recent times, some laboratory and clinical results have indicated the efficacy of MOX against ESBL-producing bacterial infections. Matsumura et al reported that treatment of bacteremia caused by ESBLproducing $E$. coli and $K$. pneumoniae with oxacephems or carbapenems resulted in a comparable outcome. ${ }^{6}$ Furthermore, time-kill studies and Monte Carlo simulation analysis have also demonstrated promising bactericidal activity of oxacephems against ESBL-producing Enterobacteriaceae. ${ }^{7,8}$ Limited pharmacokinetics (PK) and pharmacodynamics (PD) data on the activity of MOX utilized in clinics emphasizes the urgent need to evaluate the appropriate dosage regimens in killing ESBL-producing isolates. Our objective was to characterize the bacterial killing effects with simulated human exposures of cefotaxime (CTX), cefoperazone/sulbactam (CFZ/SBT), and MOX against E. coli ATCC25922 and CTXM-producing E. coli and K. pneumoniae to provide further evidence to support the clinical use of MOX in the treatment of ESBL-producing bacterial infections.

\section{Materials and methods Bacterial strains and media}

Three isolates (bla CTX-M-15 $_{15}$ positive E. coli 3376 isolated from urine, bla $\mathrm{CTX-M-14}_{\mathrm{C}}$ positive $K$. pneumoniae 2689 isolated from sputum, and E. coli ATCC25922) were investigated in this study. Organisms were grown, cultured, and quantified using Mueller-Hinton agar (MHA; \#1900515, OXOID) and Mueller-Hinton broth (\#1765821, OXOID).

\section{In vitro susceptibility testing}

MICs for all organisms were determined using the broth microdilution method, and breakpoints for resistance were defined in accordance with the CLSI. ${ }^{5}$ E. coli ATCC25922 and K. pneumoniae ATCC700603 were used as quality controls in antimicrobial susceptibility testing. It is noteworthy that the breakpoint of CFZ/SBT is consistent with the CLSI interpreted breakpoint for CFZ alone. ${ }^{9}$

\section{PK parameters employed in the study}

The simulated human serum concentrations of CTX, CFZ/ SBT, and MOX after single intravenous (IV) administration were based on PK data from previous studies (Table S1).,10-12 In the study, a 2-compartment PK model of the agents was used for all experiments. The regimens for CTX $2 \mathrm{~g}$ once daily ( 2 g qd), 2 g every 8 h ( 2 g q8h); CFZ/SBT 2 g once daily ( $2 \mathrm{~g} \mathrm{qd})$, thrice daily ( $2 \mathrm{~g} \mathrm{q} 8 \mathrm{~h}$ ); and MOX $1 \mathrm{~g}$ once daily ( $1 \mathrm{~g} \mathrm{qd})$, twice daily ( $1 \mathrm{~g} \mathrm{q} 12 \mathrm{~h})$, thrice daily $(1 \mathrm{~g} \mathrm{q} 8 \mathrm{~h}), 2 \mathrm{~g}$ once daily ( $2 \mathrm{~g} \mathrm{qd})$, twice daily ( $2 \mathrm{~g} \mathrm{q} 12 \mathrm{~h}$ ), and thrice daily ( $\mathrm{g}$ q8h) after IV administration were simulated (Figure S1).

\section{In vitro PK/PD simulation model and antibacterial activity measurements}

The in vitro PK Auto Simulation System 400 (PASS-400; Dainippon Seiki, Kyoto, Japan) utilized in this study has been described previously. ${ }^{13,14}$ Briefly, the apparatus consisted of a central unit, growth media chamber, and waste container connected by 3-way pipelines and syringe pumps to form a closed system. The central unit contained a magnetic stirrer for homogeneous mixing, and the entire unit was placed in a shaking waterbath at $37^{\circ} \mathrm{C}$. Broth medium and drug solution were pumped into the central unit via a computer-controlled peristaltic pump, while growth medium was simultaneously removed through an exit port that flowed into the waste container.

Inoculum preparation included making a bacterial suspension of $\sim 10^{8} \mathrm{CFU} / \mathrm{mL}$ from fresh subcultures of the tested isolate (incubated at $37^{\circ} \mathrm{C}$ overnight) with $0.9 \%$ normal saline. The inoculum $(1 \mathrm{~mL})$ was injected into $100 \mathrm{~mL}$ broth medium in the model to achieve the starting inoculum of $\sim 10^{6} \mathrm{CFU} / \mathrm{mL}$. Once inoculated, the strain was grown to $\log$ phase over $30 \mathrm{~min}$, and $1.5 \mathrm{~mL}$ was collected for CFU determination at predetermined time points $(0,2,4,6,10$, $14,18,20$, and $24 \mathrm{~h}$ ). Each sample was centrifuged, washed, and resuspended in normal saline thrice to avoid drug carryover effect and serially diluted in $0.9 \%$ normal saline before plating on MHA plates. Bacterial clones were counted after $18 \mathrm{~h}$ incubation. The limit of clone detection was $30 \mathrm{CFU} / \mathrm{mL}$. 
In addition, each experiment was performed in triplicate to assure reproducibility.

\section{Quantitative evaluation of the antimicrobial effect}

The PD parameters, including Maximum Kill Down (MKD, the difference between the minimum bacterial count and the initial count during the experiment), the bacterial counting difference between time 0 and $24 \mathrm{~h}\left(\Delta \log \mathrm{N}_{24}\right)$, bacterial growth recovery time (RT, the time from exposure to the antibiotic till the moment when the bacterial count again reaches its initial level), the time required for microbial initial count to be reduced by $90 \%, 99 \%, 99.9 \%\left(\mathrm{~T}_{99.9 \%}\right)$, and the time during which the concentration of the drug was over the MIC (\%T > MIC) were analyzed by PASS 400 Analyze Bactericidal Activity software (Figure S2). ${ }^{15}$ The area between the control growth curve and bactericidal curves (IE) was employed as the integral parameters for evaluating antimicrobial effect; this is preferable to other PD parameters. ${ }^{15} \mathrm{IE}$ was calculated by the trapezoidal rule using the program GraphPad Prism 6.

\section{Results}

\section{MICs of 3 agents against the strains}

Table 1 shows the MICs for CTX, CFZ, CFZ/SBT, and MOX against $E$. coli ATCC25922, 3376, and 2689. CTXM-producing isolates 3376 and 2689 were highly resistant to CTX and CFZ, while MOX resulted in low MICs $(0.5 \mathrm{mg} / \mathrm{L}$ and $0.25 \mathrm{mg} / \mathrm{L}$, respectively). In addition, MICs for CFZ/SBT against 3376 and 2689 were $16 / 16 \mathrm{mg} / \mathrm{L}$ and $32 / 32 \mathrm{mg} / \mathrm{L}$, respectively.

\section{Antibacterial effects in PK/PD simulations}

After inoculating in antibiotic-free broth, the tested strains increased from $\sim 6 \log _{10} \mathrm{CFU} / \mathrm{mL}$ to $\sim 9 \log _{10} \mathrm{CFU} / \mathrm{mL}$ at 24 $\mathrm{h}$ in the model. All tested antibiotics exhibited a rapid bactericidal efficacy against $E$. coli ATCC25922, defined as a reduction of $>4 \log _{10} \mathrm{CFU} / \mathrm{mL}$ of MKD $4 \mathrm{~h}$ after initiation of treatments, and inhibited bacterial growth $(\mathrm{RT}>24 \mathrm{~h})$ in the experimental duration (Figure 1A and B; Table 2). Notwith- standing the bacterial regrowth observed in all PK-simulated producers of CTX, CFZ/SBT, and MOX, multiple dosing ( $\mathrm{q} 8 \mathrm{~h}$ and $\mathrm{q} 12 \mathrm{~h}$ ) could inhibit the regrowth for a longer period of time than once-daily dosage regimens (Tables 2 and 3 ).

Although the presence of SBT can lower the MICs of CFZ against 3376 and 2689 from $>256 \mathrm{mg} / \mathrm{L}$ to $16 / 16 \mathrm{mg} / \mathrm{L}$ and $32 / 32 \mathrm{mg} / \mathrm{L}$, respectively, the shorter value of $\% \mathrm{~T}>\mathrm{MIC}$ achieved with CFZ/SBT ( $2 \mathrm{~g} \mathrm{qd}$ ) resulted in weak bactericidal effects, and the bacterial count was similar to that of controls at $24 \mathrm{~h}$, which was similar to that observed with CTX (2 g qd) (Table 3; Figure 1C-F). Of note, the multiple dosage regimen of CFZ/SBT ( $2 \mathrm{~g}$ q8h) also showed limited bactericidal effects and were followed by rapid regrowth against susceptible 3376 and intermediate 2689 strains. In contrast, MOX still exhibited stable antibacterial activity against both 3376 and 2689 strains, as observed in E. coli ATCC25922. The MKD and RT of MOX against ESBL-producing isolates were similar to those against E. coli ATCC25922. The multiple dosage regimens achieved larger IE than once-daily dosage regimens, especially in the simulations with $2 \mathrm{~g} \mathrm{q} 8 \mathrm{~h}$, resulting in $>145 \log _{10} \mathrm{CFU} / \mathrm{mL} \cdot \mathrm{h}^{-1}$, $>20 \mathrm{~h}$ of $\mathrm{T}_{99.9 \%}$, and $>3 \log 10$ reduction in $\mathrm{CFU} / \mathrm{mL}$ at $24 \mathrm{~h}$ against 3376 and 2689 strains (Table 2).

The relationships between $\% \mathrm{~T}>\mathrm{MIC}$ and IE are shown in Figure 2; the \% $\%$ MIC value of $50 \%$ seemed to be the lowest requirement for bacterial inhibition. The regimens of MOX (1 g q12h, $1 \mathrm{~g}$ q8h, $2 \mathrm{~g} \mathrm{q12h}$, and $2 \mathrm{~g}$ q8h) achieved $100 \%$ of $\% \mathrm{~T}>\mathrm{MIC}$ against E. coli ATCC25922, 3376, and 2689, and the IE of these simulated regimens were all over $140 \log _{10} \mathrm{CFU} / \mathrm{mL} \cdot \mathrm{h}^{-1}$. For the simulated regimens of CTX and CFZ/SBT, the shorter value of $\% \mathrm{~T}>\mathrm{MIC}$ against 3376 and 2689 resulted in smaller IE $(<10$ and $<60 \log _{10} \mathrm{CFU} / \mathrm{mL} \cdot \mathrm{h}^{-1}$, respectively), being significantly lower than the IE of MOX-simulated regimens (Table 3; Figure 2). Notably, the IE for CFZ/SBT and MOX against E. coli ATCC25922 was larger than that for tested drugs against 3376 and 2678, when \% $>$ > MIC achieved similar values, especially in the value of $50 \%$. The $70 \%$ value of $\% \mathrm{~T}>\mathrm{MIC}$ was responsible for the promising bacteriological eradication of 3376 and 2689.

Table I MICs of antibiotics against ESBL-producing bacteria and Escherichia coli ATCC25922

\begin{tabular}{lllll}
\hline Bacteria & MIC (mg/L) & & & ESBL type \\
\cline { 2 - 4 } & CTX & CFZ & CFZ/SBT (I:I) & MOX \\
\hline E. coli 3376 & $>256$ & $>256$ & $16 / 16$ & 0.5 \\
Klebsiella pneumoniae 2689 & 256 & $>256$ & $32 / 32$ & 0.25 \\
E. coli ATCC25922 & 0.06 & 0.5 & 0.5 & 0.5 \\
K. pneumoniae ATCC700603 & 256 & $>256$ & $8 / 8$ & 4 \\
\hline
\end{tabular}

Note: “"” Indicates no ESBL enzymes.

Abbreviations: ATCC, American Type Culture Collection; CFZ, cefoperazone; CTX, cefotaxime; ESBL, extended-spectrum $\beta$-lactamase; MIC, minimum inhibitory concentration; MOX, moxalactam; SBT, sulbactam. 
A

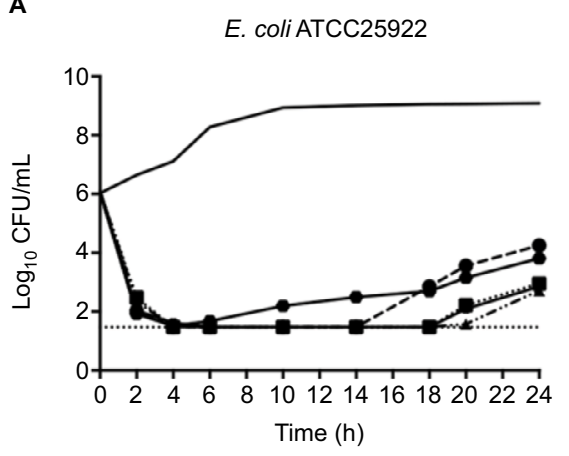

C

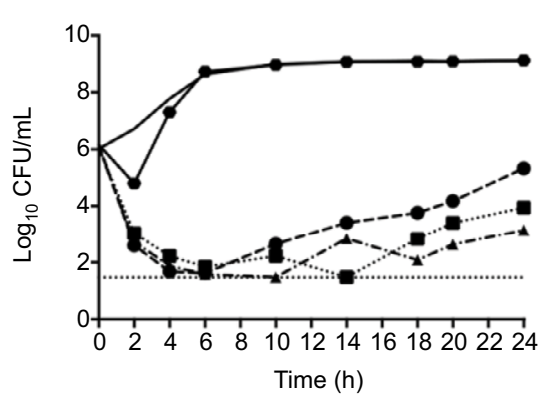

B

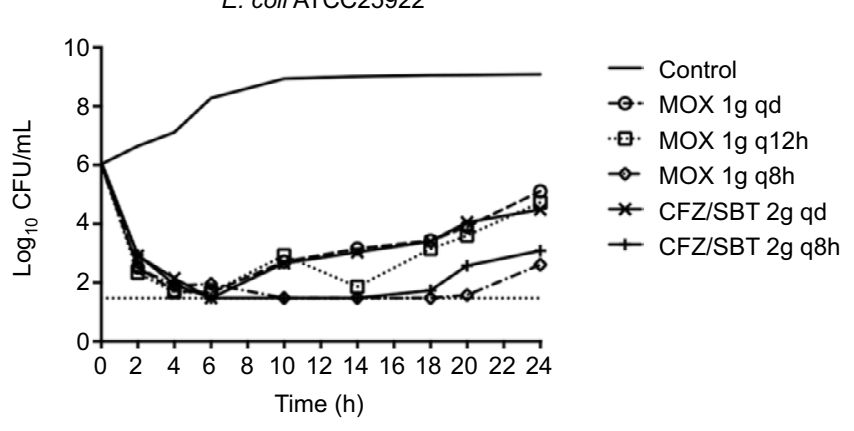

D

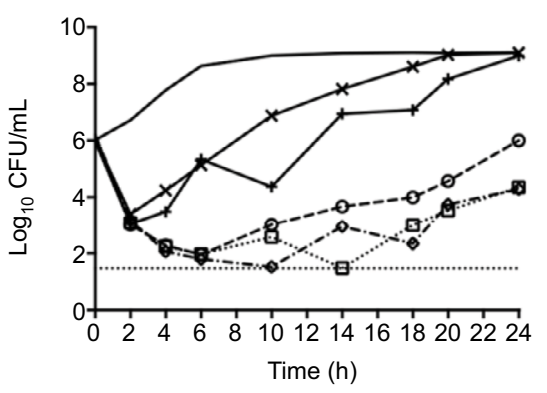

- Control

$\Theta$. MOX $1 \mathrm{~g}$ qd

๑. MOX $1 \mathrm{~g} \mathrm{q} 12 \mathrm{~h}$

$\leadsto$ MOX 1g q8h

* CFZ/SBT 2 g qd

+ CFZ/SBT $2 \mathrm{~g}$ q8h
E

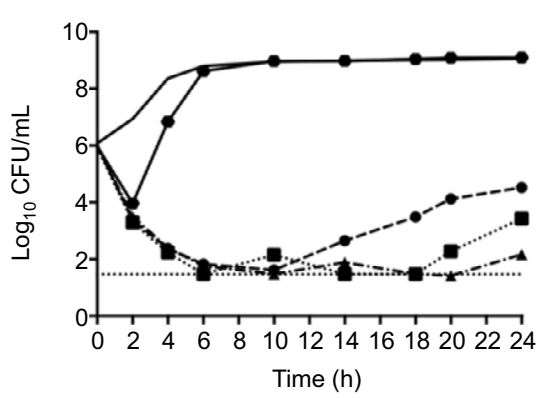

- Control

- MOX 2g q12h

- MOX 2g q8h

$\rightarrow$ CTX 2g qd

Figure I In vitro dynamic time-kill curves using human exposures of moxalactam, cefotaxime, and cefoperazone/sulbactam against Escherichia coli ATCC25922 and CTX-Mproducing E. coli and Klebsiella pneumoniae.

Notes: (A) and (B) show simulated dosing regimens against E. coli ATCC25922; (C) and (D) show simulated dosing regimens against E. coli 3376; (E) and (F) show simulated dosing regimens against $K$. pneumoniae 2689. The dotted lines indicate the regimens of MOX. The solid lines indicate the regimens of CTX and CFZ/SBT. The lower limit of detection (broken line) was $1.47 \log _{10} \mathrm{CFU} / \mathrm{mL}$.

Abbreviations: ATCC, American Type Culture Collection; CFU, colony forming units; CFZ/SBT, cefoperazone/sulbactam; CTX, cefotaxime; MOX, moxalactam; qd, oncedaily; q $2 \mathrm{~h}$, twice-daily; q8h, thrice-daily.

\section{Discussion}

ESBL-producing Enterobacteriaceae are prevalent among community- and hospital-acquired infections. ${ }^{16,17}$ Several studies have established that CTX-M-14 and CTX-M-15 are the most abundant ESBL enzymes worldwide in urinary tract, bloodstream, and intra-abdominal infections. ${ }^{18-20}$ Because antibacterial drugs for treating ESBL-producing bacterial infections are limited, carbapenems have traditionally been recommended as the drug of choice in such circumstances. However, the increased usage of carbapenems has promoted the rapid evolution and dissemination of carbapenem- and/ or multidrug-resistant isolates. ${ }^{21}$ Thus, finding alternative agents for the clinical treatment of infections caused by ESBL producers is imperative to alleviate the selective pressure on carbapenem-resistant germs.

Replacement of the structural modification of the 7-hydroxyl group in MOX makes the drug resistant to hydrolysis by ESBL enzymes. Our results indicated that MICs for MOX against 3376 and 2689 strains were $0.5 \mathrm{mg} / \mathrm{L}$ and $0.25 \mathrm{mg} / \mathrm{L}$, respectively, which was in the range of MIC val- 
Table 2 Antibacterial effect of each simulated dosing regimen of the 3 antibiotics against ESBL-producing bacteria and Escherichia coli ATCC25922

\begin{tabular}{|c|c|c|c|c|c|c|c|c|}
\hline \multicolumn{2}{|c|}{ Dosage regimen } & \multirow{2}{*}{$\begin{array}{l}\text { MKD } \\
\left(\log _{10} \text { CFU/mL) }\right. \\
-4.4\end{array}$} & \multirow{2}{*}{$\begin{array}{l}\begin{array}{l}\Delta \log N_{24} \\
\left(\log _{10} \text { CFU/mL) }\right.\end{array} \\
-0.9\end{array}$} & \multirow{2}{*}{$\begin{array}{l}\text { RT (h) } \\
>24\end{array}$} & \multirow{2}{*}{$\begin{array}{l}\mathbf{T}_{90 \%}(\mathbf{h}) \\
23.1\end{array}$} & \multirow{2}{*}{$\begin{array}{l}\mathbf{T}_{99 \%}(\mathbf{h}) \\
19.3\end{array}$} & \multirow{2}{*}{$\begin{array}{l}\mathbf{T}_{99.9 \%}(\mathbf{h}) \\
10.0\end{array}$} & \multirow{2}{*}{$\begin{array}{l}\text { IE } \\
\left(\log _{10} \mathrm{CFU} / \mathrm{mL} \mathrm{h}^{-1}\right)\end{array}$} \\
\hline 25922 & MOX (Ig qd) & & & & & & & \\
\hline & $\operatorname{MOX}(\lg q \mid 2 h)$ & -4.4 & -1.3 & $>24$ & $>23.5$ & 20.7 & 16.1 & 134.8 \\
\hline & MOX (Ig q8h) & -4.7 & -3.5 & $>24$ & $>23.5$ & $>23.0$ & $>22.5$ & 155.8 \\
\hline & MOX (2g qd) & -4.7 & -1.8 & $>24$ & $>23.6$ & 21.5 & 17.2 & 145.7 \\
\hline & $\operatorname{MOX}(2 g q \mid 2 h)$ & -4.8 & -3.2 & $>24$ & $>23.5$ & $>23.0$ & $>22.5$ & 155.4 \\
\hline & MOX (2g q8h) & -4.6 & -3.3 & $>24$ & $>23.5$ & 23.1 & $>22.5$ & I58.I \\
\hline & CTX (2g qd) & -4.6 & -2.2 & $>24$ & $>23.6$ & $>23.2$ & 18.2 & 140.8 \\
\hline & CTX (2g q8h) & -4.8 & -3.2 & $>24$ & $>23.6$ & $>23.2$ & $>22.7$ & 156.6 \\
\hline & CFZ/SBT (2g qd) & -4.6 & -1.5 & $>24$ & $>23.5$ & 18.7 & 10.0 & 128.0 \\
\hline & CFZ/SBT (2g q8h) & -4.7 & -3 & $>24$ & $>23.5$ & $>22.9$ & $>22.1$ & 151.5 \\
\hline \multirow[t]{9}{*}{3376} & MOX (Ig qd) & -4.2 & -0.2 & $>24$ & 21.1 & 17.5 & 8.5 & 118.9 \\
\hline & $\operatorname{MOX}(\lg q \mid 2 h)$ & -4.7 & -1.9 & $>24$ & $>23.5$ & 22.1 & 16.7 & 138.7 \\
\hline & MOX (Ig q8h) & -4.8 & -1.9 & $>24$ & $>23.4$ & 19.7 & 17.3 & 139.2 \\
\hline & $\operatorname{MOX}(2 g \mathrm{qd})$ & -4.6 & -0.8 & $>24$ & 23.0 & 19.0 & 10.6 & 127.7 \\
\hline & $\operatorname{MOX}(2 g q \mid 2 h)$ & -4.6 & -2.1 & $>24$ & $>23.4$ & $>22.8$ & 16.6 & 142.4 \\
\hline & MOX (2g q8h) & -4.8 & -2.9 & $>24$ & $>23.5$ & $>22.9$ & 20.6 & 148.2 \\
\hline & CTX (2g qd) & -1.4 & 3.0 & 3.2 & 1.6 & NA & NA & 45.0 \\
\hline & CFZ/SBT (2g qd) & -2.7 & 3.1 & 8.0 & 5.1 & 2.4 & NA & 39.4 \\
\hline & CFZ/SBT (2g q8h) & -3.2 & 3.0 & 12.6 & 4.9 & 3.3 & 1.1 & 62.0 \\
\hline \multirow[t]{9}{*}{2689} & MOX (Ig qd) & -3.9 & -0.5 & $>24$ & 20.3 & 14.0 & 7.3 & 116.8 \\
\hline & $\operatorname{MOX}(\lg q \mid 2 h)$ & -5.1 & -1.9 & $>24$ & $>23.3$ & 21.0 & 6.3 & 143.2 \\
\hline & MOX (Ig q8h) & -4.7 & -2 & $>24$ & $>23.3$ & $>22.5$ & 17.9 & 147.6 \\
\hline & MOX (2g qd) & -4.5 & -1.5 & $>24$ & $>23.3$ & 18.1 & 13.2 & 135.8 \\
\hline & $\operatorname{MOX}(2 g q \mid 2 h)$ & -4.7 & -2.5 & $>24$ & $>23.3$ & $>22.6$ & 19.5 & 153.0 \\
\hline & MOX (2g q8h) & -4.6 & -3.8 & $>24$ & $>23.3$ & $>22.5$ & $>21.3$ & 157.5 \\
\hline & CTX (2g qd) & -2.0 & 3.1 & 3.5 & 2.3 & 0.4 & NA & 10.5 \\
\hline & CFZ/SBT (2g qd) & -2.8 & 3.0 & 7.6 & 3.8 & 3.5 & NA & 32.4 \\
\hline & CFZ/SBT (2g q8h) & -2.7 & 3.0 & 12.0 & 4.9 & 2.8 & NA & 52.3 \\
\hline
\end{tabular}

Note: Negative numbers indicate reduction in CFU from $0 \mathrm{~h}$.

Abbreviations: CFU, colony forming units; CTX, cefotaxime; CFZ/SBT, cefoperazone/sulbactam; ESBL, extended-spectrum $\beta$-lactamase; IE, the area between the control growth and antibacterial killing curves; $\Delta \log \mathrm{N}_{24}$, the bacterial count difference between time 0 and $24 \mathrm{~h}$; MOX, moxalactam; NA, not applicable; MKD, maximum kill down; qd, once-daily; q $12 \mathrm{~h}$, twice-daily; q8h, thrice-daily; RT, bacterial growth recovery time; $\mathrm{T}_{90 \%}, \mathrm{~T}_{99 \%}$, and $\mathrm{T}_{99.9 \%}$, the time required for microbial initial count to be reduced by $90 \%, 99 \%$, and $99.9 \%$, respectively.

Table $3 \%$ T > MIC values for MOX, CTX, and CFZ/SBT treatment regimens

\begin{tabular}{|c|c|c|c|c|c|}
\hline Bacteria & $\begin{array}{l}\text { MOX } \\
\text { (Ig qd) (\%) }\end{array}$ & $\begin{array}{l}\text { MOX } \\
(\lg q \mid 2 h)(\%)\end{array}$ & $\begin{array}{l}\text { MOX } \\
\text { (Ig q8h) (\%) }\end{array}$ & $\begin{array}{l}\text { MOX } \\
\text { (2g qd) (\%) }\end{array}$ & $\begin{array}{l}\text { MOX } \\
\left(2 g q^{\prime} \mid 2 h\right)(\%)\end{array}$ \\
\hline Escherichia coli ATCC25922 & 76.60 & 100 & 100 & 85.10 & 100 \\
\hline Klebsiella pneumoniae 2689 & 87.38 & 100 & 100 & 95.63 & 100 \\
\hline E. coli 3376 & 76.60 & 100 & 100 & 85.10 & 100 \\
\hline Bacteria & $\begin{array}{l}\text { MOX } \\
\text { (2g q8h) (\%) }\end{array}$ & $\begin{array}{l}\text { CTX } \\
\text { (2g qd) (\%) }\end{array}$ & $\begin{array}{l}\text { CTX } \\
\text { (2g q8h) (\%) }\end{array}$ & $\begin{array}{l}\text { CFZ/SBT } \\
\text { (2g qd) (\%) }\end{array}$ & $\begin{array}{l}\text { CFZ/SBT } \\
\text { (2g q8h) (\%) }\end{array}$ \\
\hline E. coli ATCC25922 & 100 & 56.13 & 100 & 48.83 & 100.00 \\
\hline K. pneumoniae 2689 & 100 & 0 & 0 & 10.42 & 31.79 \\
\hline E. coli 3376 & 100 & 0 & 0 & 16.63 & 50.38 \\
\hline
\end{tabular}

Abbreviations: ATCC, American Type Culture Collection; CTX, cefotaxime; CFZ, cefoperazone; MIC, minimum inhibitory concentration; MOX, moxalactam; SBT, sulbactam; \%T > MIC, the time during which the concentration of the drug remained above the MIC; qd, once-daily; qI2h, twice-daily; q8h, thrice-daily.

ues for $90 \%$ of organisms $\left(\mathrm{MIC}_{90}\right)$ for MOX against ESBLproducing E. coli and $K$. pneumoniae $(0.5$ and $0.25 \mathrm{mg} / \mathrm{L}$, respectively) reported in a previous epidemiology antimicrobial surveillance study in China. ${ }^{22}$ MICs for CTX and CFZ/
SBT were much higher than for MOX against 3376 and 2689 Due to the powerful antibacterial activity in vitro and favorable pharmacology of MOX (longer $\mathrm{t}_{1 / 2}$, higher $\mathrm{C}_{\text {max }}$, and larger area under the curve), its clinical value is worthy of exploration. 


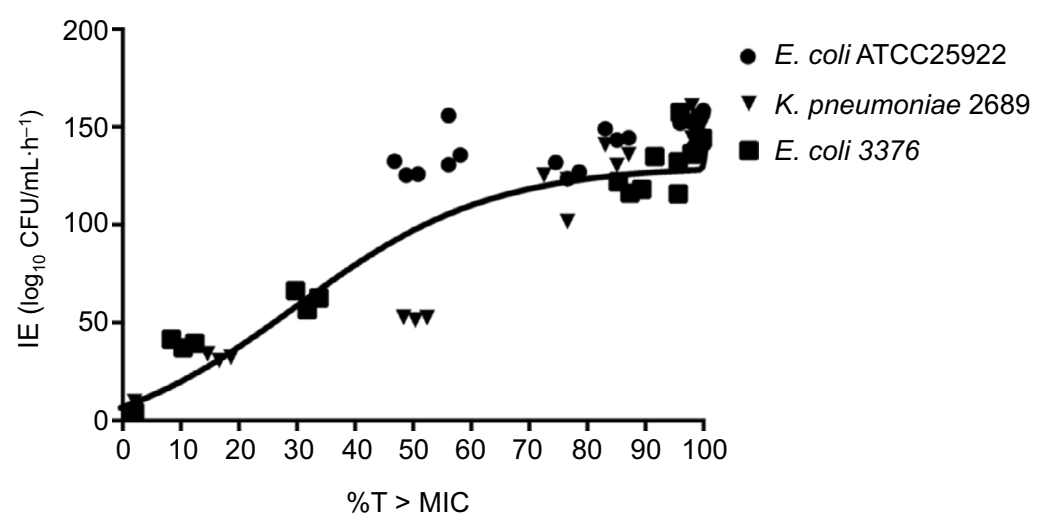

Figure 2 The relationship between \%T > MIC of MOX, CTX and CFZ/SBT against the tested bacteria and IE.

Note: Three isolates are denoted by different symbols.

Abbreviations: ATCC, American Type Culture Collection; CFU, colony forming units; CFZ, cefoperazone; CTX, cefotaxime; E. coli, Escherichia coli; IE, the area between the control growth and bacterial killing and regrowth curves; K. pneumoniae, Klebsiella pneumoniae; MIC, minimum inhibitory concentration; MOX, moxalactam; SBT, sulbactam; $\% \mathrm{~T}>\mathrm{MIC}$, the time during which the concentration of the drug remained above the MIC.

The in vitro PK/PD simulation model can simulate the human PKs of antimicrobial agents and the results can define the optimal dosage regimens to maximize clinical efficacy. Our results showed that the simulated human exposures of tested agents exhibited sustained antibacterial killing effects against non-ESBL-producing $E$. coli ATCC25922, which was also identified in an in vitro PK/PD model using ceftazidime ( $2 \mathrm{~g} \mathrm{q} 8 \mathrm{~h}$ ) against susceptible strains resulting in $\geq 3 \log _{10} \mathrm{CFU} /$ $\mathrm{mL}$ reduction from 8 to $24 \mathrm{~h} .^{23}$

It is noteworthy that MOX was more significantly bactericidal than CTX and CFZ/SBT against 3376 and 2689, presumably due to its higher stability against ESBLs and the higher $\% \mathrm{~T}>\mathrm{MIC}$ value achieved by all MOX dosing regimens. A previous study using in vitro time-kill analysis also found that flomoxef, a member of oxacephems, exhibited a rapid rate of killing (reduction of $>3 \log _{10} \mathrm{CFU} / \mathrm{mL}$ in the bacterial count at $6 \mathrm{~h}$ after application of flomoxef at $8 \mathrm{mg} / \mathrm{L}$ ) against all ESBL-producing K. pneumoniae strains, and inhibited regrowth for at least $24 \mathrm{~h} .{ }^{7}$ Subsequent clinical studies showed that treatment of bacteremia or urinary tract infections caused by ESBL-producing E. coli and $K$. pneumoniae with oxacephems or carbapenems resulted in a similar outcome. In a multicenter retrospective study of 126 patients with bacteremia caused due to ESBL-producing $E$. coli, no difference was found in adjusted clinical success between those treated with flomoxef or cefmetazole $(1 \mathrm{~g} \mathrm{q} 8 \mathrm{~h})$ and carbapenems $(1 \mathrm{~g}$ q $8 \mathrm{~h}$ or $0.5 \mathrm{~g} \mathrm{q} 8 \mathrm{~h}$ ) at 30 days (empirical therapy, $100 \%$ versus $96.5 \%$; definitive therapy, $98.4 \%$ versus $96.3 \%$ ). ${ }^{6}$ These results highlighted the promising efficacy of MOX for the treatment of infections caused by ESBL-producing isolates.

Given that $\% \mathrm{~T}>\mathrm{MIC}$ is the optimal PD index for $\beta$-lactams, increasing the free drug concentration above the MIC should be a major aim of treatment, which was observed by higher \% $>$ MIC and larger IE achieved for MOX ( $1 \mathrm{~g}$ q12h) compared with MOX (2 g qd). Notably, the target value of $\% \mathrm{~T}>\mathrm{MIC}$ for treating ESBL producers was found to be more stringent than non-ESBL-producing isolates with MOX and $\mathrm{CFZ} / \mathrm{SBT}$, similar to a previous study showing reduced efficacy for piperacillin/tazobactam against ESBL-producing $K$. pneumoniae compared with non-ESBL-producing K. pneumoniae at identical drug exposure (Figure 2). ${ }^{24}$ And $>70 \%$ of $\% \mathrm{~T}>\mathrm{MIC}$ was required for the promising bacterial killing of ESBL producers in this study. Therefore, physicians should use multiple-dosing regimens and prolonged and continuous infusion of $\beta$-lactams to achieve a higher $\% \mathrm{~T}>$ MIC when treating ESBL-producing isolates with MICs that are relatively high but still within the range of susceptibility.

$\beta$-lactam/ $\beta$-lactamase inhibitor combinations (BLBLIs) account for a large proportion of antibiotic consumption in China. CFZ/SBT is the second most widely used combination in hospitals, and is recommended for the treatment of infections caused by ESBL producers in clinics..$^{25,26}$ However, several disadvantages for using BLBLIs to treat ESBL-producing isolates have been reported. The size of the inoculum can affect results, as can the presence of other $\beta$-lactam resistance genes such as $b l a_{C M Y-2}$ and $b l a_{F O X-5}$, as well as decreased membrane permeability. ${ }^{27-29}$ Moreover, PK/PD studies indicate that conventional dosing regimens for BLBLIs cannot achieve the target $\% \mathrm{~T}>\mathrm{MIC}$ value associated with positive outcomes (especially when the MIC is at the higher end of susceptible range).$^{30}$ In the present study, CFZ/SBT failed to maintain effective killing and allowed significant regrowth to occur, as shown by the bacterial count, which was similar to untreated controls at $24 \mathrm{~h}$. The $\% \mathrm{~T}>\mathrm{MIC}$ value against $E$. coli 3376 and $K$. pneumoniae 2689 was low at all tested doses. However, CFZ/SBT (2 g q8h) also displayed 
poor antibacterial activity against $E$. coli 3376 when $\% \mathrm{~T}$ $>$ MIC exceeded $50 \%$. This may be because the exposureresponse relationship may be different between drug classes, species, and host immune response. Indeed, McKinnon et al suggested that patients receiving cefepime or ceftazidime for the treatment of serious infections with $\% \mathrm{~T}>\mathrm{MIC}$ of $100 \%$ had a significantly greater chance of a clinical cure $(82 \%$ versus $33 \%)$ and bacterial eradication (97\% versus $44 \%$ ) than patients with $\% \mathrm{~T}>\mathrm{MIC}$ of $<100 \% .{ }^{31}$ Although authors of a prospective controlled clinical trial from October 2002 to April 2005 did show a favorable efficacy for CFZ/SBT ( $2 \mathrm{~g}$ q8h) in the treatment of infections caused by ESBL-producing E. coli coupled with clinical treatment success rates of $71.4 \%$, this could be because all isolates tested were susceptible to CFZ/SBT and patients were clinically stable..$^{32}$ Nevertheless, it should be noted that resistance of ESBL-producing isolates to BLBLIs is on the rise, especially in developing countries. Recent epidemiological studies in China reported high prevalence of ESBL producers in community-onset bloodstream infections and $\mathrm{MIC}_{90}$ values for ESBL-producing $E$. coli and $K$. pneumoniae with CFZ/SBT of $64 \mathrm{mg} / \mathrm{L} .{ }^{22,33}$ Thus, the empirical therapy of CFZ/SBT ( $2 \mathrm{~g} \mathrm{q} 8 \mathrm{~h}$ ) inevitably decreases the clinical efficacy, especially for severely ill patients, and this combination of agents should be used with more caution.

The application of preclinical PD studies using dynamic PK/PD models can provide more representative data for in vivo bacterial killing situation. Although our in vitro $\mathrm{PK} / \mathrm{PD}$ simulation failed to account for the host immune response, the presence of a competent immune system can markedly reduce the required PD values. ${ }^{34}$ Thus, the bacterial killing effects of MOX against ESBL producers may be more encouraging in animal experiments and clinical trials. The next step should focus on current regimens of MOX against ESBL producers with animal models to design more effective and representative therapies.

\section{Conclusion}

The need for effective therapeutic agents to combat infections caused by ESBL-producing isolates is widely known, and access to antimicrobial drugs continues to be limited. Our in vitro PK/PD simulation model demonstrated superior bactericidal activity of MOX under conventional dosing regimens against ESBL-producing E. coli and $K$. pneumoniae. The simulated regimen of CFZ/SBT, which is often prescribed by clinicians for the treatment of infections in China at present, showed limited bactericidal effects against ESBL producers. The findings of this study are supportive in prescribing MOX as an alternative agent against ESBL producers in clinical practice. Further studies, including in vitro tests, animal models, and clinical trials, are required to evaluate the exact efficacy of MOX as a reasonable carbapenem-sparing option for the treatment of infections caused by ESBL producers.

\section{Acknowledgments}

We thank Whiteson Pharma for providing the drugs MOX, CTX, and CFZ/SBT. This work was supported by the Key Research and Development Program of Zhejiang (no 2015C03032) and the National Basic Research Program of China (973 program, no 2015CB554,201).

\section{Author contributions}

$\mathrm{YX}, \mathrm{CH}$ and $\mathrm{BZ}$ developed the concept and designed the experiments. CH, WY, TN, TX and JZ performed the experiments. BZ and YX gave conceptual advice. $\mathrm{CH}$ wrote the paper. All authors contributed toward data analysis, drafting and revising the paper and agree to be accountable for all aspects of the work.

\section{Disclosure}

The authors report no conflicts of interest in this work.

\section{References}

1. Organization WH. Antimicrobial resistance: global report on surveillance. Australas Med J. 2014;7(4):237.

2. Pitout JD, Laupland KB. Extended-spectrum beta-lactamase-producing Enterobacteriaceae: an emerging public-health concern. Lancet Infect Dis. 2008;8(3):159-166.

3. Timsit JF, Pilmis B, Zahar JR. How should we treat hospital-acquired and ventilator-associated pneumonia caused by extended-spectrum beta-lactamase-producing enterobacteriaceae? Semin Respir Crit Care Med. 2017;38(3):287-300.

4. Carmine AA, Brogden RN, Heel RC, Romankiewicz JA, Speight TM, Avery GS. Moxalactam (latamoxef). A review of its antibacterial activity, pharmacokinetic properties and therapeutic use. Drugs. 1983;26(4):279-333.

5. Franklin R, Breamrouwenhorst Matthew A, Karen B, Michael N, George M, Dwigh TJ. Performance Standards for Antimicrobial Susceptibility Testing; 26th International Supplement. 2016. Available from: http:// www.clsi.org/. Accessed December 21, 2017.

6. Matsumura Y, Yamamoto M, Nagao M, et al. Multicenter retrospective study of cefmetazole and flomoxef for treatment of extended-spectrum-beta-lactamase-producing Escherichia coli bacteremia. Antimicrob Agents Chemother. 2015;59(9):5107-5113.

7. Lee CH, Su LH, Tang YF, Liu JW. Treatment of ESBL-producing Klebsiella pneumoniae bacteraemia with carbapenems or flomoxef: a retrospective study and laboratory analysis of the isolates. JAntimicrob Chemother. 2006;58(5):1074-1077.

8. Ito A, Tatsumi Y, Wajima T, Nakamura R, Tsuji M. Potent antibacterial activities of latamoxef (moxalactam) against ESBL producing Enterobacteriaceae analyzed by Monte Carlo simulation. Jpn J Antibiot. 2014;67(2):109-122.

9. Jones RN, Barry AL, Packer RR, Gregory WW, Thornsberry C. In vitro antimicrobial spectrum, occurrence of synergy, and recommendations for dilution susceptibility testing concentrations of the cefoperazonesulbactam combination. J Clin Microbiol. 1987;25(9):1725-1729. 
10. Vallee F, LeBel M. Comparative study of pharmacokinetics and serum bactericidal activity of ceftizoxime and cefotaxime. Antimicrob Agents Chemother. 1991;35(10):2057-2064.

11. Drusano GL, Standiford HC, Fitzpatrick B, et al. Comparison of the pharmacokinetics of ceftazidime and moxalactam and their microbiological correlates in volunteers. Antimicrob Agents Chemother. 1984;26(3):388-393.

12. Wei Min-ji, Zhao Cai-yun, QI Hui-min, et al. The pharmacokinetic study of cefoperazone/sulbactam (1:1) in adults and the elderly. Chin J Clin Pharmacol. 2007;23(1):28-32.

13. Oonishi Y, Mitsuyama J, Yamaguchi K. Effect of GrlA mutation on the development of quinolone resistance in Staphylococcus aureus in an in vitro pharmacokinetic model. $J$ Antimicrob Chemother. 2007;60(5):1030-1037.

14. Yu W, Zhou K, Guo L, et al. In vitro pharmacokinetics/pharmacodynamics evaluation of fosfomycin combined with amikacin or colistin against KPC2-producing Klebsiella pneumoniae. Front Cell Infect Microbiol. 2017;7:246.

15. Firsov AA, Vostrov SN, Shevchenko AA, Cornaglia G. Parameters of bacterial killing and regrowth kinetics and antimicrobial effect examined in terms of area under the concentration-time curve relationships: action of ciprofloxacin against Escherichia coli in an in vitro dynamic model. Antimicrob Agents Chemother. 1997;41(6):1281-1287.

16. Singh N, Pattnaik D, Neogi DK, Jena J, Mallick B. Prevalence of ESBL in Escherichia coli Isolates Among ICU Patients in a Tertiary Care Hospital. J Clin Diagn Res. 2016;10(9):DC19-DC22.

17. Castillo-Tokumori F, Irey-Salgado C, Malaga G. Worrisome high frequency of extended-spectrum beta-lactamase-producing Escherichia coli in community-acquired urinary tract infections: a case-control study. Int J Infect Dis. 2016;55:16-19.

18. Koksal I, Yilmaz G, Unal S, et al. Epidemiology and susceptibility of pathogens from SMART 2011-12 Turkey: evaluation of hospitalacquired versus community-acquired urinary tract infections and ICUversus non-ICU-associated intra-abdominal infections. J Antimicrob Chemother. 2017;72(5):1364-1372.

19. Bou-Antoun S, Davies J, Guy R, Johnson AP, Sheridan EA, Hope RJ. Descriptive epidemiology of Escherichia coli bacteraemia in England, April 2012 to March 2014. Euro Surveill. 2016;21(35).

20. Flores-Mireles AL, Walker JN, Caparon M, Hultgren SJ. Urinary tract infections: epidemiology, mechanisms of infection and treatment options. Nat Rev Microbiol. 2015;13(5):269-284.

21. Chia JH, Su LH, Lee MH, et al. Development of high-level carbapenem resistance in Klebsiella pneumoniae among patients with prolonged hospitalization and carbapenem exposure. Microb Drug Resist. 2010;16(4):317-325.

22. Yang Q, Zhang H, Cheng J, et al. In vitro activity of flomoxef and comparators against Escherichia coli, Klebsiella pneumoniae and Proteus mirabilis producing extended-spectrum beta-lactamases in China. Int J Antimicrob Agents. 2015;45(5):485-490.
23. Alou L, Aguilar L, Sevillano D, et al. Is there a pharmacodynamic need for the use of continuous versus intermittent infusion with ceftazidime against $P$ seudomonas aeruginosa? An in vitro pharmacodynamic model. J Antimicrob Chemother. 2005;55(2):209-213.

24. Burgess DS, Hall RG. 2nd. In vitro killing of parenteral beta-lactams against standard and high inocula of extended-spectrum beta-lactamase and non-ESBL producing Klebsiella pneumoniae. Diagn Microbiol Infect Dis. 2004:49(1):41-46.

25. Harris PN, Tambyah PA, Paterson DL. Beta-lactam and beta-lactamase inhibitor combinations in the treatment of extended-spectrum betalactamase producing Enterobacteriaceae: time for a reappraisal in the era of few antibiotic options? Lancet Infect Dis. 2015;15(4): 475-485.

26. Guo H, Qin J, Xiang J. Surveillance for and susceptibility of Acinetobacter baumannii in a large hospital and burn center in Shanghai, China, 2007-2013. Am J Infect Control. 2016;44(12):1718-1719.

27. Liu X, Thungrat K, Boothe DM. Occurrence of OXA- 48 carbapenemase and other beta-lactamase genes in ESBL-producing multidrug resistant Escherichia coli from dogs and cats in the United States, 2009-2013. Front Microbiol. 2016;7:1057.

28. Bradford PA, Urban C, Mariano N, Projan SJ, Rahal JJ, Bush K. Imipenem resistance in Klebsiella pneumoniae is associated with the combination of ACT-1, a plasmid-mediated AmpC beta-lactamase, and the foss of an outer membrane protein. Antimicrob Agents Chemother. 1997;41(3):563-569.

29. Thomson KS, Moland ES. Cefepime, piperacillin-tazobactam, and the inoculum effect in tests with extended-spectrum beta-lactamaseproducing Enterobacteriaceae. Antimicrob Agents Chemother. 2001;45(12):3548-3554.

30. Ambrose PG, Bhavnani SM, Jones RN. Pharmacokinetics-pharmacodynamics of cefepime and piperacillin-tazobactam against Escherichia coli and Klebsiella pneumoniae strains producing extended-spectrum beta-lactamases: report from the ARREST program. Antimicrob Agents Chemother. 2003;47(5):1643-1646.

31. McKinnon PS, Paladino JA, Schentag JJ. Evaluation of area under the inhibitory curve (AUIC) and time above the minimum inhibitory concentration ( $\mathrm{T}>\mathrm{MIC}$ ) as predictors of outcome for cefepime and ceftazidime in serious bacterial infections. Int JAntimicrob Agents. 2008;31(4):345-351.

32. Bin C, Hui W, Renyuan Z, et al. Outcome of cephalosporin treatment of bacteremia due to CTX-M-type extended-spectrum beta-lactamase-producing Escherichia coli. Diagn Microbiol Infect Dis. 2006;56(4):351-357.

33. Quan J, Zhao D, Liu L, et al. High prevalence of ESBL-producing Escherichia coli and Klebsiella pneumoniae in community-onset bloodstream infections in China. J Antimicrob Chemother. 2017;72(1):273-280.

34. Nicasio AM, Crandon JL, Nicolau DP. In vivo pharmacodynamic profile of tigecycline against phenotypically diverse Escherichia coli and Klebsiella pneumoniae isolates. Antimicrob Agents Chemother. 2009;53(7):2756-2761. 


\section{Supplementary materials}

A

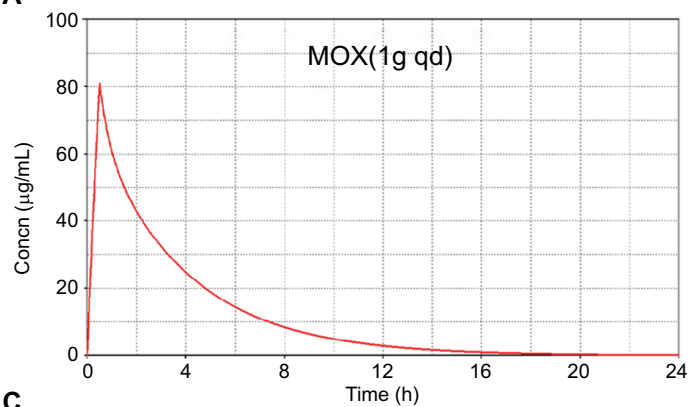

C

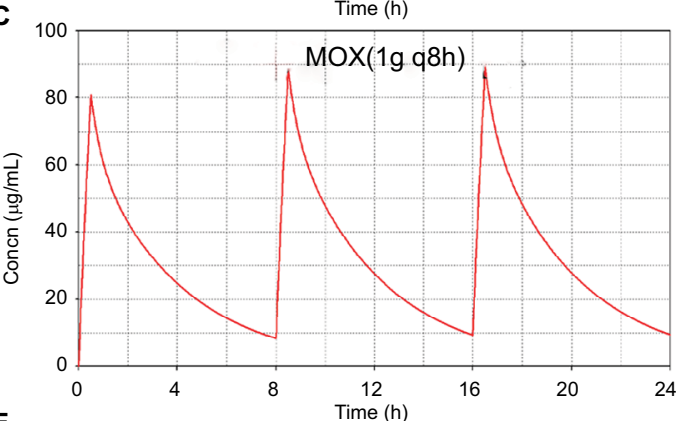

$\mathbf{E}$

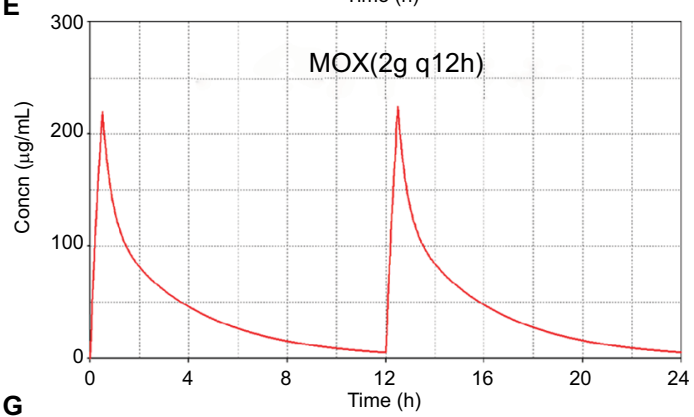

G
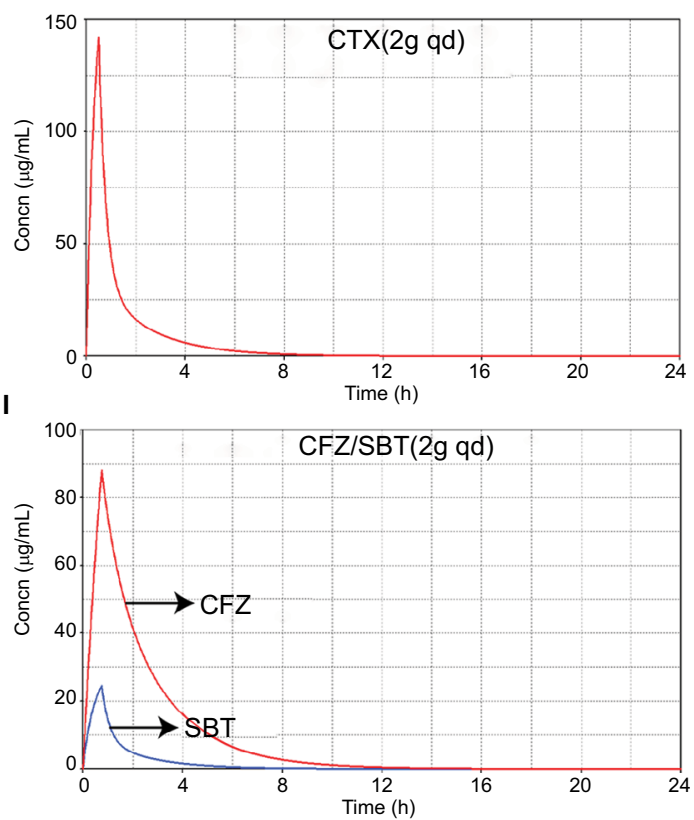
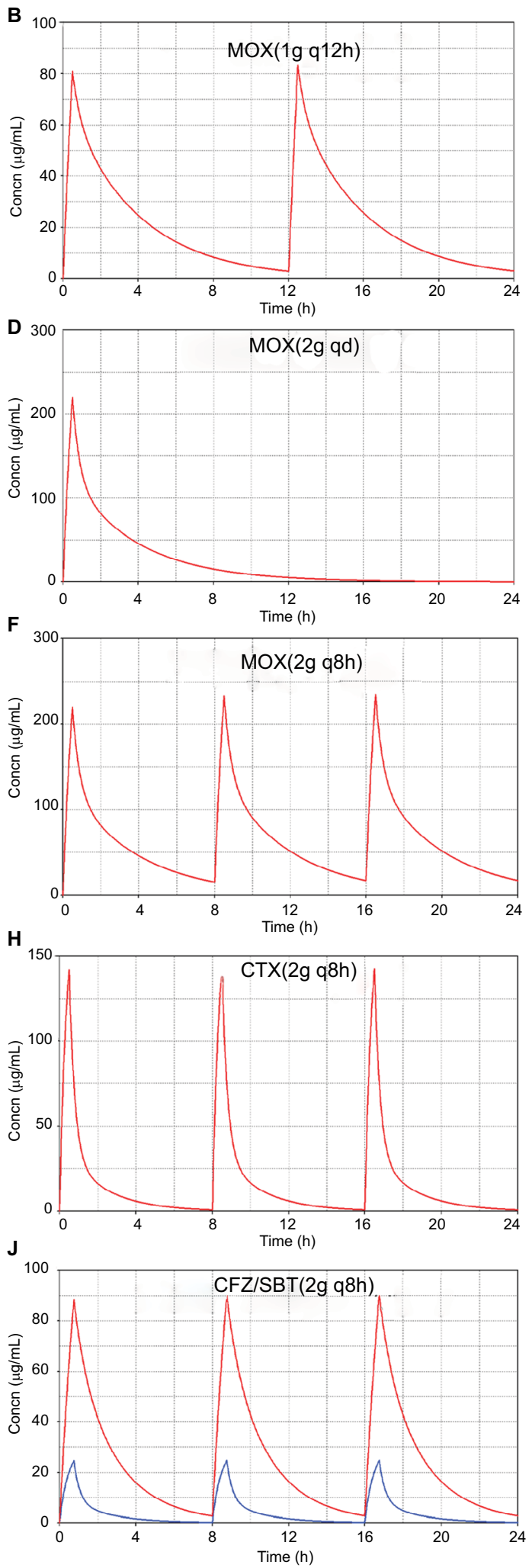

Figure SI The simulated time-concentration curve of each dosage regimen in the in vitro Pharmacokinetics Auto Simulation System 400.

Notes: (A), (B), and (C) show simulated time-concentration curve of MOX at Ig qd, Ig q I $2 \mathrm{~h}$, and Ig q8h, respectively; (D), (E), and (F) show simulated time-concentration curve of MOX at $2 \mathrm{~g} q d, 2 \mathrm{~g} q \mid 2 \mathrm{~h}$, and $2 \mathrm{~g} q 8 \mathrm{~h}$, respectively; $(\mathbf{G})$ and $(\mathbf{H})$ show simulated time-concentration curve of CTX at $2 \mathrm{~g}$ qd and $2 \mathrm{~g} q 8 \mathrm{~h}$, respectively; (I) and $(\mathrm{J})$ show simulated time-concentration curve of CFZ/SBT at $2 \mathrm{~g}$ qd and $2 \mathrm{~g} q 8 \mathrm{~h}$, respectively.

Abbreviations: CFZ, cefoperazone; CTX, cefotaxime; MOX, moxalactam; qd, once-daily; ql2h, twice-daily; q8h, thrice-daily; SBT, sulbactam. 


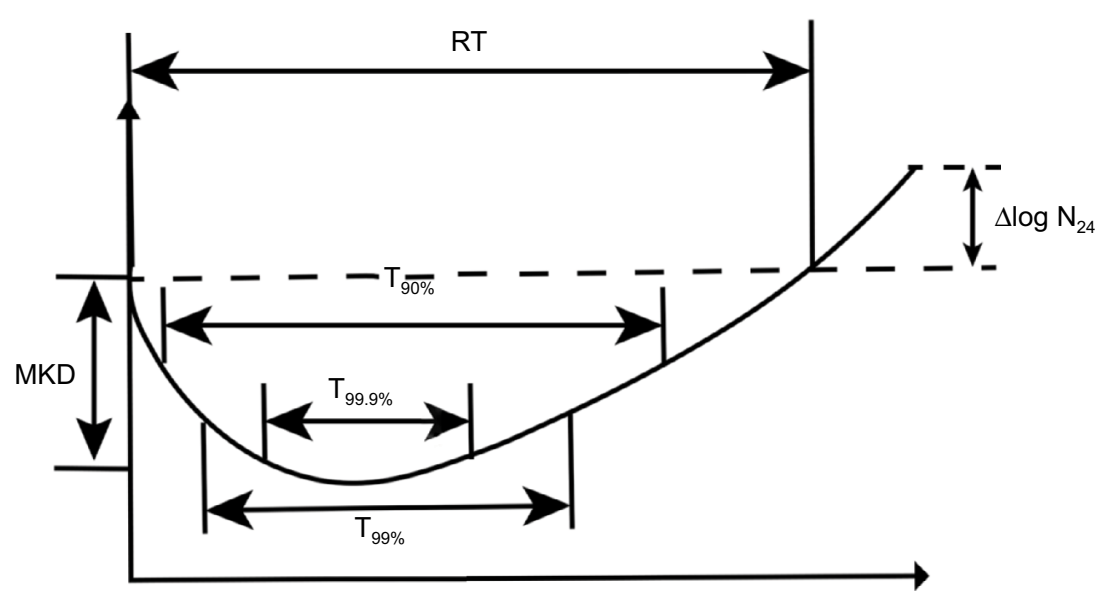

Figure S2 The sketch of pharmacodynamic parameters.

Abbreviations: MKD, the difference between the minimum bacterial count and the initial count during the experiment; $\Delta$ log $\mathrm{N}_{24}$, the bacterial count difference between time 0 and 24 h; RT, bacterial growth recovery time, the time from exposure to antibiotic till the moment when the bacterial count again reaches its initial level; $\mathrm{T}_{90 \%}, \mathrm{~T}_{90 \%}$ and $\mathrm{T}_{99.9 \%}$, the time required for microbial initial count to be reduced by $90 \%, 99 \%, 99.9 \%$, respectively.

Table SI The pharmacokinetic parameters employed in the study of CTX, CFZ/SBT, and MOX after intravenous infusion

\begin{tabular}{lllllll}
\hline Drug and dosage & Infusion time $(\mathbf{m i n})$ & $\mathbf{C}_{\max }(\boldsymbol{\mu} / \mathbf{m L})$ & $\mathbf{T}_{1 / 2 \alpha}(\mathbf{h})$ & $\mathbf{T}_{1 / 2 \beta}(\mathbf{h})$ & $\mathbf{A U C}(\boldsymbol{\mu} \cdot \mathbf{h} / \mathbf{m L})$ & References \\
\hline CTX $2 \mathrm{~g}$ & 30 & 125.86 & 0.2 & 1.43 & 141.93 & $\mathrm{I}$ \\
MOX 2g & 30 & 219.8 & 0.26 & 2.52 & 536 & 2 \\
MOX Ig & 30 & 81 & 0.28 & 2.56 & 264 & 3 \\
CFZ/SBT 2g (I:I) & 45 (CFZ) & 88.33 & 0.43 & 1.55 & 200.4 & 4 \\
& 45 (SBT) & 24.53 & 0.21 & 1.3 & 32.08 & 4 \\
\hline
\end{tabular}

Abbreviations: $\mathrm{C}_{\max }$, peak serum concentration; $\mathrm{T}_{1 / 2 \alpha}$, distribution half-life; $\mathrm{T}_{1 / 2 \beta}$, elimination half-life; AUC, area under the concentration-time curve. MOX, moxalactam; CTX, cefotaxime; CFZ, cefoperazone; SBT, sulbactam.

\section{References}

1. Oonishi Y, Mitsuyama J, Yamaguchi K. Effect of GrlA mutation on the development of quinolone resistance in Staphylococcus aureus in an in vitro pharmacokinetic model. JAntimicrob Chemother. 2007;60(5):1030-1037.

2. Yu W, Zhou K, Guo L, et al. In vitro pharmacokinetics/pharmacodynamics evaluation of fosfomycin combined with amikacin or colistin against KPC2-producing Klebsiella pneumoniae. Front Cell Infect Microbiol. 2017;7:246.
3. Firsov AA, Vostrov SN, Shevchenko AA, Cornaglia G. Parameters of bacterial killing and regrowth kinetics and antimicrobial effect examined in terms of area under the concentration-time curve relationships: action of ciprofloxacin against Escherichia coli in an in vitro dynamic model. Antimicrob Agents Chemother. 1997;41(6):1281-1287.

4. Singh N, Pattnaik D, Neogi DK, Jena J, Mallick B. Prevalence of ESBL in Escherichia coli Isolates Among ICU Patients in a Tertiary Care Hospital. J Clin Diagn Res. 2016;10(9):DC19-DC22.
Infection and Drug Resistance

\section{Publish your work in this journal}

Infection and Drug Resistance is an international, peer-reviewed openaccess journal that focuses on the optimal treatment of infection (bacterial, fungal and viral) and the development and institution of preventive strategies to minimize the development and spread of resistance. The journal is specifically concerned with the epidemiology of antibiotic resistance and the mechanisms of resistance development and diffusion in both hospitals and the community. The manuscript management system is completely online and includes a very quick and fair peerreview system, which is all easy to use. Visit http://www.dovepress.com/ testimonials.php to read real quotes from published authors. 